\title{
Characterizations of smooth spaces by approximate orthogonalities
}

\author{
PAWEŁ WÓJCIK
}

\begin{abstract}
Since the monograph by Amir that appeared in 1986, a lot of attention has been given to the problem of characterizing, by means of properties of the norms, when a Banach space is indeed a Hilbert space, i.e., when the norm derives from an inner product. In this paper, similar investigations will be carried out for smooth spaces instead of inner product spaces. We consider the approximate orthogonalities in real normed spaces. We show that the relations approximate semi-orthogonality and approximate $\rho_{+}$-orthogonality are generally incomparable (unless the normed space is smooth). As a result, we give a characterization of smooth spaces in terms of those approximate orthogonalities.
\end{abstract}

Mathematics Subject Classifications. Primary 46B20; 46C50; Secondary; 46C99.

Keywords. Approximate orthogonality, Smoothness, Norm derivative, Semi-inner product.

\section{Introduction}

Semi-inner products and norm derivatives, which can be naturally defined in general normed spaces over the real number field, play an essential role in describing the geometric properties of these spaces. The aim of this paper is to provide a complete overview of characterizations of smooth normed linear spaces based on norm derivatives.

In a real normed space, one can define various orthogonality relations. In the paper, we will consider the Birkhoff-James orthogonality:

$$
x \perp_{\mathrm{B}} y: \Leftrightarrow \forall_{\lambda \in \mathbb{R}}\|x\| \leqslant\|x+\lambda y\| .
$$

An approximate Birkhoff orthogonality was given by Chmieliński [3]:

$$
x \perp_{\mathrm{B}}^{\varepsilon} y: \Leftrightarrow \forall_{\lambda \in \mathbb{R}}\|x\|^{2} \leqslant\|x+\lambda y\|^{2}+2 \varepsilon\|x\| \cdot\|\lambda y\| .
$$

Obviously, for $\varepsilon=0$ we have $\perp_{\mathrm{B}}=\perp_{\mathrm{B}}^{\varepsilon}$.

We recall some of the main properties of the semi-inner product. Let $(X,\|\cdot\|)$ be a normed space over $\mathbb{K} \in\{\mathbb{R}, \mathbb{C}\}$. A mapping $[\cdot \mid \cdot]: X \times X \rightarrow \mathbb{K}$ will be called a semi-inner product (briefly s.i.p.), if the following properties are satisfied: 
(sip1) $\forall_{x, y, z \in X} \forall_{\alpha, \beta \in \mathbb{K}} \quad[\alpha x+\beta y \mid z]=\alpha[x \mid z]+\beta[y \mid z] ;$

(sip2) $\forall_{x, y \in X} \forall_{\alpha \in \mathbb{K}} \quad[x \mid \alpha y]=\bar{\alpha}[x \mid y]$;

(sip3) $\forall_{x \in X} \quad[x \mid x]=\|x\|^{2}$;

(sip4) $\forall x, y \in X \quad|[x \mid y]| \leqslant\|x\| \cdot\|y\|$.

Lumer [9] and Giles [7] showed that in each normed space a semi-inner product always exists, however, not necessarily a unique one. There exists a unique s.i.p. in $X$ if and only if $X$ is smooth. Now, for a fixed s.i.p. in $X$ we define a semi-orthogonality, by

$$
x \perp_{\mathrm{s}} y: \Leftrightarrow[y \mid x]=0 .
$$

We introduce $\varepsilon$-s-orthogonality

$$
x \perp_{\mathrm{s}}^{\varepsilon} y: \Leftrightarrow|[y \mid x]| \leqslant \varepsilon\|y\| \cdot\|x\| .
$$

From this moment, throughout this paper, all normed spaces are assumed to be over reals and at least two-dimensional. Let $(X,\|\cdot\|)$ be a real normed space. We define two mappings $\rho_{+}^{\prime}, \rho_{-}^{\prime}: X \times X \rightarrow \mathbb{R}$ :

$$
\rho_{ \pm}^{\prime}(x, y):=\lim _{t \rightarrow 0^{ \pm}} \frac{\|x+t y\|^{2}-\|x\|^{2}}{2 t}=\|x\| \cdot \lim _{t \rightarrow 0^{ \pm}} \frac{\|x+t y\|-\|x\|}{t} .
$$

These mappings are called norm derivatives. Now, we recall their useful properties (the proofs can be found in $[2,6]$ ):

(nd1) $\forall_{x, y \in X} \forall_{\alpha \in \mathbb{R}} \quad \rho_{ \pm}^{\prime}(x, \alpha x+y)=\alpha\|x\|^{2}+\rho_{ \pm}^{\prime}(x, y)$;

(nd2) $\forall_{x, y \in X} \quad \forall_{\alpha \geqslant 0} \quad \rho_{ \pm}^{\prime}(\alpha x, y)=\alpha \rho_{ \pm}^{\prime}(x, y)=\rho_{ \pm}^{\prime}(x, \alpha y)$;

(nd2') $\forall_{x, y \in X} \forall_{\alpha<0} \quad \rho_{ \pm}^{\prime}(\alpha x, y)=\alpha \rho_{\mp}^{\prime}(x, y)=\rho_{ \pm}^{\prime}(x, \alpha y)$;

(nd3) $\forall_{x \in X} \quad \rho_{ \pm}^{\prime}(x, x)=\|x\|^{2}$;

(nd4) $\forall x, y \in X \quad\left|\rho_{ \pm}^{\prime}(x, y)\right| \leqslant\|x\| \cdot\|y\|$.

Moreover, the mappings $\rho_{+}^{\prime}, \rho_{-}^{\prime}$ are continuous with respect to the second variable, but not necessarily with respect to the first one.

Now, fix the semi-inner product $[\cdot \cdot \cdot]$. Then,

$$
\forall_{x, y \in X} \quad \rho_{-}^{\prime}(x, y) \leqslant[y \mid x] \leqslant \rho_{+}^{\prime}(x, y) .
$$

It is known that, $X$ is smooth if and only if $\rho_{+}^{\prime}(x, y)=\rho_{-}^{\prime}(x, y)=[y \mid x]$ for all $x, y \in X$.

The following mapping $\rho^{\prime}: X \times X \rightarrow \mathbb{R}$ was introduced by Miličić [10]:

$$
\rho^{\prime}(x, y):=\frac{1}{2}\left(\rho_{+}^{\prime}(x, y)+\rho_{-}^{\prime}(x, y)\right)
$$

and is called an M-semi-inner product (briefly $M$-s.i.p.).

In a similar way as earlier, we define $\varepsilon$ - $\rho_{+}$-orthogonality, $\varepsilon-\rho_{-}$-orthogonality and $\varepsilon$ - $\rho$-orthogonality:

$$
\begin{aligned}
x \perp_{\rho_{+}}^{\varepsilon} y & : \Leftrightarrow\left|\rho_{+}^{\prime}(x, y)\right| \leqslant \varepsilon\|x\| \cdot\|y\|, \\
x \perp_{\rho_{-}}^{\varepsilon} y & : \Leftrightarrow\left|\rho_{-}^{\prime}(x, y)\right| \leqslant \varepsilon\|x\| \cdot\|y\|, \\
x \perp_{\rho}^{\varepsilon} y & : \Leftrightarrow \quad\left|\rho^{\prime}(x, y)\right| \leqslant \varepsilon\|x\| \cdot\|y\| .
\end{aligned}
$$


It is obvious that we define $\perp_{\rho_{+}}:=\perp_{\rho_{+}}^{\varepsilon}$ with $\varepsilon:=0$. For the orthogonalities $\perp_{\rho_{-}}, \perp_{\rho}$ the definitions are similar.

The relations $\perp_{\rho_{ \pm}}^{\varepsilon}$ and $\perp_{\mathrm{B}}^{\varepsilon}$ are generally comparable. It is proved in $[5$, Theorem 3.2] that for an arbitrary real normed space $X$ we have

$$
\perp_{\rho_{+}}^{\varepsilon} \subset \perp_{\mathrm{B}}^{\varepsilon}, \quad \perp_{\rho_{-}}^{\varepsilon} \subset \perp_{\mathrm{B}}^{\varepsilon}, \quad \perp_{\rho}^{\varepsilon} \subset \perp_{\mathrm{B}}^{\varepsilon}, \quad \perp_{\mathrm{s}}^{\varepsilon} \subset \perp_{\mathrm{B}}^{\varepsilon} .
$$

If $(X,\langle\cdot \mid \cdot\rangle)$ is an inner product space, then $\langle x \mid y\rangle=[y \mid x]=\rho_{+}^{\prime}(x, y)=\rho_{-}^{\prime}(x, y)$ $=\rho^{\prime}(x, y)$ for arbitrary $x, y \in X$. Hence we have $\perp^{\varepsilon}=\perp_{\mathrm{s}}^{\varepsilon}=\perp_{\rho_{+}}^{\varepsilon}=\perp_{\rho_{-}}^{\varepsilon}=$ $\perp_{\rho}^{\varepsilon}=\perp_{\mathrm{B}}^{\varepsilon}$.

\section{Comparison of $\varepsilon-\rho_{ \pm}$-orthogonality and $\varepsilon$-s-orthogonality}

R. C. James (see [8]) introduced in a real normed space $X$ the following orthogonality relation: $x \perp_{\mathrm{J}} y: \Leftrightarrow\|x+y\|=\|x-y\|$. Amir [1] showed that $\perp_{\mathrm{J}} \subset \perp_{\mathrm{B}}$ or $\perp_{\mathrm{J}} \supset \perp_{\mathrm{B}}$ if and only if the norm in X comes from an inner product. Similar investigations will be carried out for smooth spaces instead of inner product spaces.

Relations $\perp_{\rho_{+}}^{\varepsilon}, \perp_{\rho_{-}}^{\varepsilon}$ and $\perp_{\rho}^{\varepsilon}$ are generally (unless $X$ is smooth) incomparable (cf. [5, Theorem 3.3]). Relations $\perp_{\rho}^{\varepsilon}, \perp_{\mathrm{s}}^{\varepsilon}$ are also incomparable (cf. [5, Theorem 3.5]). Now, let us compare the approximate orthogonalities $\perp_{\mathrm{s}}^{\varepsilon}$ and $\perp_{\rho_{ \pm}}^{\varepsilon}$. The next theorem is the main result of the paper.

Theorem 1. Let $X$ be a real normed space and let $[\cdot \cdot \cdot]$ be a fixed semi inner product in $X$. Then the following conditions are equivalent.
(i) $\perp_{\rho_{+}}^{\varepsilon} \subset \perp_{\mathrm{s}}^{\varepsilon}$
(ii) $\perp_{\rho_{+}}^{\varepsilon} \supset \perp_{\mathrm{s}}^{\varepsilon}$
(iii) $\perp_{\rho_{+}}^{\varepsilon}=\perp_{\mathrm{s}}^{\varepsilon}$
(iv) $\perp_{\rho_{-}}^{\varepsilon} \subset \perp_{\mathrm{s}}^{\varepsilon}$
(v) $\perp_{\rho_{-}}^{\varepsilon} \supset \perp_{\mathrm{s}}^{\varepsilon}$
(vi) $\perp_{\rho_{-}}^{\varepsilon}=\perp_{\mathrm{s}}^{\varepsilon}$
(vii) $X$ is smooth.

Proof. We start with proving (i) $\Rightarrow$ (vii). Fix arbitrarily two linearly independent vectors $x, y \in X$ such that $\|x\|=\|y\|=1$. First, suppose that $\rho_{+}^{\prime}(x, y)=-\varepsilon$. Then $x \perp_{\rho_{+}}^{\varepsilon} y$. Using (i), we get $x \perp_{\mathrm{s}}^{\varepsilon} y$, which means $|[y \mid x]| \leqslant \varepsilon$. This inequality, together with $-\varepsilon \leqslant[y \mid x] \stackrel{(1)}{\leqslant} \rho_{+}^{\prime}(x, y)=-\varepsilon$, yields $\rho_{+}^{\prime}(x, y)=[y \mid x]$.

Next, suppose that $\rho_{+}^{\prime}(x, y)<-\varepsilon$ Then we define a mapping $\varphi:[0,1] \rightarrow \mathbb{R}$ by $\varphi(t):=\rho_{+}^{\prime}\left(x, \frac{t x+(1-t) y}{\|t x+(1-t) y\|}\right)$. Since $\varphi$ is continuous, $\varphi(0)=\rho_{+}^{\prime}(x, y)<-\varepsilon$ and $\varphi(1)=1$, it follows that there exists $t_{o} \in(0,1)$ such that $\varphi\left(t_{o}\right)=-\varepsilon$.

Since $\varphi\left(t_{o}\right)=-\varepsilon$, defining $\alpha:=\frac{t_{o}}{\left\|t_{o} x+\left(1-t_{o}\right) y\right\|}, \quad \beta:=\frac{\left(1-t_{o}\right)}{\left\|t_{o} x+\left(1-t_{o}\right) y\right\|} \quad$ and $\quad z:=\alpha x+\beta y$ we get $\|z\|=1$ and $\rho_{+}^{\prime}(x, z)=-\varepsilon$, thus $x \perp_{\rho_{+}}^{\varepsilon} z$. Moreover, we have $\beta>0$. 
Using (i), we get $x \perp_{\mathrm{s}}^{\varepsilon} z$, which means $|[z \mid x]| \leqslant \varepsilon$. This inequality, together with $-\varepsilon \leqslant[z \mid x] \stackrel{(1)}{\leqslant} \rho_{+}^{\prime}(x, z)=-\varepsilon$, yields

$$
\rho_{+}^{\prime}(x, z)=[z \mid x] .
$$

Applying properties of functionals $\rho_{+}^{\prime}$ and $[\cdot \mid \cdot]$ we get

$$
\begin{aligned}
\rho_{+}^{\prime}(x, y) & =\rho_{+}^{\prime}\left(x,-\frac{\alpha}{\beta} x+\frac{1}{\beta} z\right) \stackrel{(\mathrm{nd} 1)}{=}-\frac{\alpha}{\beta}\|x\|^{2}+\rho_{+}^{\prime}\left(x, \frac{1}{\beta} z\right) \stackrel{(\mathrm{nd} 2)}{=} \\
& =-\frac{\alpha}{\beta}\|x\|^{2}+\frac{1}{\beta} \rho_{+}^{\prime}(x, z) \stackrel{(3)}{=}-\frac{\alpha}{\beta}\|x\|^{2}+\frac{1}{\beta}[z \mid x] \stackrel{(\text { sip } 1)}{=} \\
& =\left[-\frac{\alpha}{\beta} x+\frac{1}{\beta} z \mid x\right]=[y \mid x] .
\end{aligned}
$$

(In case $\rho_{+}^{\prime}(x, y)>-\varepsilon$, we define $\psi:[0,1] \rightarrow \mathbb{R}$ by $\psi(t):=\rho_{+}^{\prime}\left(x, \frac{t(-x)+(1-t) y}{\|t(-x)+(1-t) y\|}\right)$ and show that with some $t_{o} \in(0,1), \psi\left(t_{o}\right)=-\varepsilon$. Then we define

$\alpha:=\frac{-t_{o}}{\left\|t_{o}(-x)+\left(1-t_{o}\right) y\right\|}, \quad \beta:=\frac{\left(1-t_{o}\right)}{\left\|t_{o}(-x)+\left(1-t_{o}\right) y\right\|} \quad$ and $\quad z:=\alpha x+\beta y$ to obtain $x \perp_{\rho_{+}}^{\varepsilon} y$. The rest runs similarly.)

We have shown that functionals $\rho_{+}^{\prime}(\cdot, \diamond)$ and $[\diamond \mid \cdot]$ are equal on unit, linearly independent vectors $x, y$. Now, let $x, y \in X$ be arbitrary vectors. If $y=\gamma x$, then $\rho_{+}^{\prime}(x, y)=\gamma\|x\|^{2}=[y \mid x]$. Next, suppose that $x, y$ are linearly independent. Then we get

$$
\rho_{+}^{\prime}(x, y) \stackrel{(\mathrm{nd} 2)}{=}\|x\| \cdot\|y\| \rho_{+}^{\prime}\left(\frac{x}{\|x\|}, \frac{y}{\|y\|}\right)=\|x\| \cdot\|y\|\left[\frac{y}{\|y\|} \mid \frac{x}{\|x\|}\right] \stackrel{\left(\operatorname{sip}^{2}\right)}{=}[y \mid x] .
$$

We have shown that functionals $\rho_{+}^{\prime}(\cdot, \diamond)$ and $[\diamond \mid \cdot]$ are equal. Then we have

$$
\rho_{-}^{\prime}(x, y) \stackrel{\left(\text { nd2 }^{\prime}\right)}{=}-\rho_{+}^{\prime}(x,-y)=-[-y \mid x] \stackrel{(\text { sip } 1)}{=}[y \mid x]=\rho_{+}^{\prime}(x, y) .
$$

We have proved that functionals $\rho_{-}^{\prime}$ and $\rho_{+}^{\prime}$ are equal. Thus $X$ is smooth.

Implication (ii) $\Rightarrow$ (vii) can be shown similarly. In the second part of the above proof one should consider (sip1) instead of (nd1). Similarly, we can consider (nd1) instead of ( $\operatorname{sip} 1)$. Next, we may interchange the role of (sip2) and (nd2) (or (nd2')) in the above reasoning. Implications (vii) $\Rightarrow$ (iii), (iii) $\Rightarrow$ (i) and (iii) $\Rightarrow$ (ii) are obvious. Therefore, we get (i) $\Leftrightarrow($ iii $) \Leftrightarrow($ vii) and (ii) $\Leftrightarrow$ (iii) $\Leftrightarrow($ vii).

In the same manner, one can prove (iv) $\Leftrightarrow(\mathrm{vi}) \Leftrightarrow(\mathrm{vii})$ and (v) $\Leftrightarrow(\mathrm{vi}) \Leftrightarrow($ vii).

\section{Comparison of $\varepsilon$ - $B$-orthogonality and $\varepsilon$-s-orthogonality}

Fix arbitrarily $\varepsilon \in[0,1)$. Chmieliński proved that $\perp_{\mathrm{s}}^{\varepsilon} \subset \perp_{\mathrm{B}}^{\varepsilon}$ (cf. [3, Proposition 3.1]). In the general case, we may get $\perp_{\mathrm{B}}^{\varepsilon} \nsubseteq \perp_{\mathrm{s}}^{\varepsilon}$ (see [3, Example 3.1]. Moreover, 
he proved that if $X$ is a smooth space, then $\perp_{\mathrm{B}}^{\varepsilon} \subset \perp_{\mathrm{s}}^{\varepsilon}$ and $\perp_{\mathrm{B}}^{\varepsilon}=\perp_{\mathrm{s}}^{\varepsilon}$ (cf. [3, Proposition 3.2, Theorem 3.3]).

The problem arises whether the reverse is true. Namely, whether $\perp_{\mathrm{B}}^{\varepsilon} \subset \perp_{\mathrm{s}}^{\varepsilon}$ implies the smoothness of $X$. We show that this problem has an affirmative answer for real spaces.

Theorem 2. Let $X$ be a real normed space and let $\varepsilon \in[0,1)$. Then the following conditions are equivalent.
(a) $\perp_{\mathrm{B}}^{\varepsilon} \subset \perp_{\mathrm{s}}^{\varepsilon}$
(b) $\perp_{\mathrm{B}}^{\varepsilon}=\perp_{\mathrm{s}}^{\varepsilon}$
(c) $X$ is smooth.

Proof. It follows from [3, Theorem 3.3]) that $(\mathrm{c}) \Rightarrow(\mathrm{b})$ holds. The implication (b) $\Rightarrow$ (a) is obvious. We show (a) $\Rightarrow(\mathrm{c})$. Assume that $\perp_{\mathrm{B}}^{\varepsilon} \subset \perp_{\mathrm{s}}^{\varepsilon}$. Applying (2) we have $\perp_{\rho_{+}}^{\varepsilon} \subset \perp_{\mathrm{B}}^{\varepsilon}$. Thus, we obtain $\perp_{\rho_{+}}^{\varepsilon} \subset \perp_{\mathrm{s}}^{\varepsilon}$. It follows from Theorem 1 ((i) $\Leftrightarrow($ vii $))$ that $X$ is smooth.

The proof for the case $\varepsilon=0$ (i.e., $\perp_{\mathrm{B}} \subset \perp_{\mathrm{s}}$ implies the smoothness) can be found in [6, p.155, Theorem 65].

Open Access. This article is distributed under the terms of the Creative Commons Attribution License which permits any use, distribution, and reproduction in any medium, provided the original author(s) and the source are credited.

\section{References}

[1] Amir, D.: Characterization of Inner Product Spaces. Birkhäuser, Basel (1986)

[2] Alsina, C., Sikorska, J., Santos Tomás, M.: Norm Derivatives and Characterizations of Inner Product Spaces. World Scientific, Hackensack (2009)

[3] Chmieliński, J.: On an $\varepsilon$-Birkhoff orthogonality. J. Inequal. Pure Appl. Math. 6(3), Art. 79 (2005)

[4] Chmieliński, J., Wójcik, P.: On a $\rho$-orthogonality. Aequationes Math. 80, 45-55 (2010)

[5] Chmieliński, J., Wójcik, P.: $\rho$-orthogonality and its preservation-revisited. In: Recent Developments in Functional Equation and Inequalities, Banach Center Publications, vol. 99, pp. 17-30. Institute of Mathematic, Polish Academy of Sciences, Warszawa (2013)

[6] Dragomir, S.S.: Semi-Inner Products and Applications. Nova Science Publishers, Inc., Hauppauge (2004)

[7] Giles, J.R.: Classes of semi-inner-product spaces. Trans. Am. Math. Soc. 129, 436$446(1967)$

[8] James, R.C.: Orthogonality in normed linear spaces. Duke Math. J. 12, 291-302 (1945)

[9] Lumer, G.: Semi-inner-product spaces. Trans. Am. Math. Soc. 100, 29-43 (1961)

[10] Miličić, P.M.: Sur la G-orthogonalité dans les espaces normés. Mat. Vesnik 39, 325$334(1987)$ 
P. Wójcik

Institute of Mathematics

Pedagogical University of Cracow

Podchorążych 2

30-084 Kraków

Poland

e-mail: pwojcik@up.krakow.pl

Received: March 13, 2014

Revised: June 23, 2014 